\title{
POSITIVE MASS THEOREM FOR THE PANEITZ-BRANSON OPERATOR
}

\author{
EMMANUEL HUMBERT AND SIMON RAULOT
}

\begin{abstract}
We prove that under suitable assumptions, the constant term in the Green function of the Paneitz-Branson operator on a compact Riemannian manifold $(M, g)$ is positive unless $(M, g)$ is conformally diffeomophic to the standard sphere. The proof is inspired by the positive mass theorem on spin manifolds by Ammann-Humbert AH03.
\end{abstract}

\section{INTRODUCTION}

Let $(M, g)$ be a compact Riemannian manifold of dimension $n \geq 4$. We denote by $Q_{g}$ the $Q$-curvature for the metric $g$ defined by

$$
Q_{g}:=\frac{n^{2}-4}{8 n(n-1)^{2}} S_{g}^{2}-\frac{2}{(n-2)^{2}}\left|E_{g}\right|^{2}+\frac{1}{2(n-1)} \Delta_{g} S_{g},
$$

where $\Delta_{g}=-\operatorname{div}_{g} \nabla$ is the Laplace-Beltrami operator, $S_{g}$ stands for the scalar curvature of $g,\left|E_{g}\right|$ denotes the $g$-norm of the Einstein tensor $E_{g}:=\mathrm{Ric}_{g}-\frac{S_{g}}{n} g$ and $\mathrm{Ric}_{g}$ is the Ricci curvature of $g$. The Paneitz-Branson operator introduced for $n=4$ by Paneitz in Pa83 and whose definition was generalized in dimension greater than 5 by Branson [Br87, is defined for all $u \in C^{\infty}(M)$ by

$$
P_{g} u:=\Delta_{g}^{2} u-\operatorname{div}_{g}\left(A_{g} d u\right)+\frac{n-4}{2} Q_{g} u
$$

where

$$
A_{g}:=\frac{(n-2)^{2}+4}{2(n-1)(n-2)} S_{g} g-\frac{4}{n-2} \operatorname{Ric}_{g} .
$$

This operator is closely related to the problem of prescribing $Q$-curvature in a conformal class as well as the Yamabe operator (see (5) below) is related to the problem of prescribing the scalar curvature in a conformal class. It is a conformally covariant operator in the sense that if $g^{\prime}=e^{2 f} g$ is conformal to $g$, then for all $v \in C^{\infty}(M)$,

$$
P_{g^{\prime}}\left(e^{-\frac{n-4}{2} f} v\right)=e^{-\frac{n+4}{2} f} P_{g}(v) .
$$

In particular, if $n \geq 5$, and if we set $u=e^{\frac{n-4}{2} f}$ so that $g^{\prime}=u^{\frac{4}{n-4}} g$, we get for all $v \in C^{\infty}(M)$

$$
P_{g^{\prime}}\left(u^{-1} v\right)=u^{-\frac{n+4}{n-4}} P_{g}(v) .
$$

From now on, we make the following assumptions:

(a) $g$ is conformally flat;

(b) $n \geq 5$;

Key words and phrases. Paneitz-Branson operator, positive mass theorem. 
(c) the Yamabe invariant is positive (see for instance [Au98 or He97]) i.e. $g$ is conformal to a metric $g^{\prime}$ for which the scalar curvature is positive.

(d) the operator $P_{g}$ is positive.

Under Assumptions (a) to (d), it is well known that the Green's function $G_{g}$ of $P_{g}$ exists, is unique and smooth on $M \backslash\{p\}$. By the conformal convariance of the Paneitz-Branson operator, if $g^{\prime}=u^{\frac{4}{n-4}} g$ is conformal to $g$, then

$$
G_{g^{\prime}}(x, y)=\frac{G_{g}(x, y)}{u(x) u(y)} .
$$

Now, let $p \in M$. By (1), up to a conformal change of metric, we can assume

(a') $g$ is flat around $p$.

Then, it is known that we have the following expansion when $x$ is close to $p$,

$$
G_{g}(x, p)=\frac{1}{2(n-2)(n-4) \omega_{n-1} d_{g}(x, p)^{n-4}}+A+\alpha_{p}(x)
$$

where $\omega_{n-1}$ stands for the volume of the $(n-1)$-dimensional sphere, $A \in \mathbb{R}, \alpha_{p}$ is a smooth function defined around $p$ and satisfying $\alpha_{p}(p)=0$. By analogy to the case of the conformal Laplacian (see again [Au98, He97]), the number $A$ is called the mass of the Paneitz-Branson operator. If $g^{\prime}=u^{\frac{4}{n-4}} g$ is another metric conformal to $g$ and flat around $p$, then the mass $A^{\prime}$ corresponding to the metric $g^{\prime}$ is given by

$$
A^{\prime}=\frac{A}{u(p)^{2}} \text {. }
$$

Hence, the mass $A$ depends on the choice of the metric in the conformal class, but not its sign. This is the reason why in the statement of Theorem 1.1 below, we do not need to assume $\left(\mathbf{a}^{\prime}\right)$.

We also make the following assumption

(e) $G_{g}>0$ on $M \backslash\{p\}$.

For interesting results concerning Assumptions (d) and (e), the reader may refer to Grunau-Robert GR07.

The main result of the paper is the following:

Theorem 1.1. Under assumptions (a) to (e), the mass A satisfies

$$
A \geq 0
$$

with equality if and only if $(M, g)$ is conformally diffeomorphic to the sphere.

Theorem 1.1 has been already proven with the additional assumption that the Poincaré exponent is small enough (see QR06a, QR06b). In this case, Qing and Raske proved also the positivity of the Green's function of $G_{g}$.

Our proof is inspired from the positive mass theorem on spin manifolds by AmmannHumbert in AH03 (see also Raulot Ra07). The difficulty here is to overcome 
the fact that on non-spin manifolds, there is no equivalent of the SchrödingerLichnerowicz Formula.

Hebey and Robert proved the nice following result which is an analogue for geometric equations of order 4 of a hard problem concerning the Yamabe Equation:

Theorem (Hebey, Robert; [HR04]). Let $(M, g)$ be a conformally flat compact manifold of dimension $n \geq 5$. Assume $g$ has a positive Yamabe invariant, that $P_{g}$ is positive as well as its Green function and that the mass of $P_{g}$ is positive. Then, the geometric equation

$$
P_{g} u=u^{\frac{n+4}{n-4}}
$$

is compact.

In particular, together with Theorem 1.1, we get rid of the positivity of the mass.

Acknoledgements We want to thank Emmanuel Hebey and Frédéric Robert who gave us many helpful informations and references on the subject.

\section{Proof of Theorem 1.1}

In the whole proof, we can work with Assumption $\left(\mathbf{a}^{\prime}\right)$ which does not restrict the generality as explained above. To avoid complicated formulas, we set

$$
H(x)=2(n-2)(n-4) \omega_{n-1} G_{g}(x, p) .
$$

By Relation (2), $H$ satisfies the following expansion near $p$

$$
H(x)=\frac{1}{d_{g}(x, p)^{n-4}}+B+\alpha(x)
$$

where $B=2(n-2)(n-4) \omega_{n-1} A$ and where $\alpha=2(n-2)(n-4) \omega_{n-1} \alpha_{p}$ is smooth around $p$ and satisfies $\alpha(p)=0$. Theorem 1.1 is equivalent to show that $B \geq 0$ with equality if and only if $(M, g)$ is conformally diffeomorphic to the standard sphere. For any metric $g$, let

$$
L_{g}:=\frac{4(n-1)}{n-2} \Delta_{g}+S_{g}
$$

be the Yamabe operator. We recall some well known facts about $L_{g}$. The reader may refer to [Au98, He97 for further informations. First, as well as $P_{g}, L_{g}$ is conformally covariant. If $g^{\prime}=u^{\frac{4}{n-2}} g$ is conformal to $g$ then

$$
L_{g^{\prime}}\left(u^{-1} \cdot\right)=u^{-\frac{n+2}{n-2}} L_{g}(\cdot)
$$

It follows that the scalar curvatures $S_{g}$ and $S_{g^{\prime}}$ are related by the following equation

$$
L_{g} u=S_{g^{\prime}} u^{\frac{n+2}{n-2}} .
$$

By Assumptions $\left(\mathbf{a}^{\prime}\right)$ and $(\mathbf{b})$, the Green's function $\Lambda_{g}$ of $L_{g}$ exists, is unique, smooth and positive on $M \backslash\{p\}$. Setting $\Gamma(x)=4(n-1) \omega_{n-1} \Lambda_{g}(x, p)$ to simplify formulas, we have when $x$ is close to $p$

$$
\Gamma(x)=\frac{1}{d_{g}(x, p)^{n-2}}+C+\beta(x)
$$


where by $C \in \mathbb{R}, \beta$ is a smooth function defined around $p$ and satisfies $\beta(0)=0$. We define a new metric $g^{\prime}:=\Gamma^{\frac{4}{n-2}} g$ conformal to $g$ on $M_{0}:=M \backslash\{p\}$. Then, by (5)

$$
S_{g^{\prime}}=\Gamma^{-\frac{n+2}{n-2}} L_{g}(\Gamma) \equiv 0
$$

on $M_{0}$. We set $H^{\prime}=\Gamma^{-\frac{n-4}{n-2}} H$. By conformal covariance of the Paneitz-Branson operator (11) and since $P_{g} H=0$ on $M_{0}$, we have $P_{g^{\prime}} H^{\prime} \equiv 0$ on $M_{0}$. Define for all $\epsilon>0$ small enough, $M_{\epsilon}:=M \backslash B^{g}(p, \epsilon)$ where $B^{g}(p, \epsilon)$ stands for the ball of center $p$ and radius $\epsilon$ with respect to the metric $g$. We have

$$
\int_{M_{\epsilon}} P_{g^{\prime}} H^{\prime} d v_{g^{\prime}}=0 \text {. }
$$

By Relation (7) and from the definition of $P_{g}$ we have

$$
P_{g^{\prime}} H^{\prime}=\Delta_{g^{\prime}}^{2} H^{\prime}-\operatorname{div}_{g^{\prime}}\left(\frac{4}{n-2} \operatorname{Ric}_{g^{\prime}} d H^{\prime}\right)-\frac{n-4}{(n-2)^{2}}\left|E_{g^{\prime}}\right|^{2} H^{\prime} .
$$

Set $S_{\epsilon}:=\partial M_{\epsilon}=\partial B^{g}(p, \epsilon)$ be the $(n-1)$-dimensional sphere of center $p$ and radius $\epsilon$. We let $d s_{g^{\prime}}$ (resp. $d s_{g}$ ) be the volume element induced by $g^{\prime}$ (resp. g) on $S_{\epsilon}$. Integrating by part the above relation, we obtain

$$
\int_{M_{\epsilon}} P_{g^{\prime}} H^{\prime} d v_{g^{\prime}}=-\mathbf{I}+\frac{4}{n-2} \mathbf{I I}-\frac{1}{2} \int_{M_{\epsilon}}\left|E_{g^{\prime}}\right|^{2} H^{\prime} d v_{g^{\prime}}
$$

where

$$
\left\{\begin{array}{ccc}
\mathbf{I} & = & \int_{S_{\epsilon}} \partial_{\nu^{\prime}} \Delta_{g^{\prime}} H^{\prime} d s_{g^{\prime}} \\
\mathbf{I I} & = & \int_{S_{\epsilon}} \operatorname{Ric}_{g^{\prime}}\left(\operatorname{grad}^{g^{\prime}} H^{\prime}, \nu^{\prime}\right) d s_{g^{\prime}} .
\end{array}\right.
$$

Here, $\nu^{\prime}$ denotes the unit outer normal vector on $S_{\epsilon}=\partial M_{\epsilon}$ with respect to the metric $g^{\prime}$.

2.1. Computation of I. First, we notice that the scalar curvatures $S_{g}$ and $S_{g^{\prime}}$ vanish on $S_{\epsilon}$. For $g$, this comes from Assumption $\left(\mathbf{a}^{\prime}\right)$ and for $g^{\prime}$, this follows from (77). Consequently, using Formula (44) and

$$
\begin{aligned}
\Delta_{g^{\prime}} H^{\prime} & =\frac{n-2}{4(n-1)} L_{g^{\prime}} H^{\prime} \\
& =\frac{n-2}{4(n-1)} \Gamma^{-\frac{n+2}{n-2}} L_{g}\left(\Gamma H^{\prime}\right) \\
& =\frac{n-2}{4(n-1)} \Gamma^{-\frac{n+2}{n-2}} L_{g}\left(\Gamma^{\frac{2}{n-2}} H\right)
\end{aligned}
$$

We obtain

$$
\Delta_{g^{\prime}} H^{\prime}=\Gamma^{-\frac{n+2}{n-2}} \Delta_{g}\left(\Gamma^{\frac{2}{n-2}} H\right) .
$$

We set $r:=d_{g}(x, p)$. From Formulas (3) and (6), we have:

$$
\Gamma^{\frac{2}{n-2}} H=\left(\frac{1}{r^{n-2}}+C+\beta(x)\right)^{\frac{2}{n-2}}\left(\frac{1}{r^{n-4}}+B+\alpha(x)\right) .
$$

Then, using Taylor formula at $p$,

$$
\Gamma^{\frac{2}{n-2}} H=r^{2-n}+B r^{-2}+O\left(r^{-1}\right)
$$


where in the whole proof, $O\left(r^{m}\right)$ denotes a smooth function defined in a neighborhood of $p$ and which satisfies

$$
\left|\nabla_{g}^{k} O\left(r^{m}\right)\right|_{g} \leq C_{k} r^{m-k}
$$

for all $k \in \mathbb{N}$. Since $g$ is flat around $p$, we have for radially symmetric functions $f$,

$$
\Delta_{g} f(r)=-f^{\prime \prime}(r)-\frac{n-1}{r} f^{\prime}(r)
$$

Hence, this gives that near $p$,

$$
\Delta_{g} \Gamma^{\frac{2}{n-2}} H=2(n-4) B r^{-4}+O\left(r^{-3}\right)
$$

and hence by (10) and (6)

$$
\Delta_{g^{\prime}} H^{\prime}=\Gamma^{-\frac{n+2}{n-2}} \Delta_{g} H=2(n-4) B r^{n-2}+O\left(r^{n-1}\right) .
$$

We then obtain

$$
\frac{\partial}{\partial r}\left(\Delta_{g^{\prime}} H^{\prime}\right)=2(n-2)(n-4) B r^{n-3}+O\left(r^{n-2}\right) .
$$

On $S_{\epsilon}, r \equiv \epsilon$. In addition,

$$
\nu^{\prime}=-\Gamma^{-\frac{2}{n-2}} \frac{\partial}{\partial r}=-\left(\epsilon^{2}+o\left(\epsilon^{2}\right)\right) \frac{\partial}{\partial r}
$$

and

$$
d s_{g^{\prime}}=\Gamma^{2 \frac{n-1}{n-2}} d s_{g}=\Gamma^{2 \frac{n-1}{n-2}} \epsilon^{n-1} d s=\left(\epsilon^{1-n}+o\left(\epsilon^{1-n}\right)\right) d s .
$$

where $d s$ stands for the standard volume element on the unit $(n-1)$-sphere. By Formulas (12), (13) and (14), we obtain

$$
\mathbf{I}=-2(n-2)(n-4) \omega_{n-1} B+o(1)
$$

2.2. Computation of II. If $g^{\prime}=e^{2 f} g$ is conformal to $g$, then the following formula holds (see [He97] p. 240 or [Au98]):

$$
\operatorname{Ric}_{g^{\prime}}=\operatorname{Ric}_{g}-(n-2) \nabla^{2} f+(n-2) \nabla f \otimes \nabla f+\left(\Delta_{g} f-(n-2)|\nabla f|_{g}^{2}\right) g .
$$

In this context, $f=\frac{2}{n-2} \log (\Gamma)$. By (6), we have near $p$

$$
\begin{aligned}
f & =\frac{2}{n-2} \log \left(\frac{1}{r^{n-2}}+O(1)\right) \\
& =-2 \log (r)+O\left(r^{n-2}\right) .
\end{aligned}
$$

Let $\left(r, \Theta_{1}, \cdots, \Theta_{n-1}\right)$ be polar coordinates on $\mathbb{R}^{n}$. The Christoffel symbols $\Gamma_{r, \Theta_{i}}^{r}$ of the Euclidean metric in these coordinates identically vanish. This implies that for any radially symmetric function $h$, the mixed terms $\nabla_{r \Theta_{i}}^{2} h$ are zero. Since $g$ is flat near $p$, we deduce that

$$
\nabla^{2} f=\frac{2}{r^{2}} d r^{2}+b+\bar{O}\left(r^{n-4}\right)
$$

where, as in what follows, we denote by $\bar{O}\left(r^{m}\right)$ a 2-form whose norm with respect to $g$ is $O\left(r^{m}\right)$ and where $b$ is a 2 -form such that

$$
b\left(\cdot, \frac{\partial}{\partial r}\right) \equiv 0
$$


Using (11), one also computes that

$$
\begin{aligned}
\nabla f \otimes \nabla f & =\frac{4}{r^{2}} d r^{2}+\bar{O}\left(r^{n-4}\right) \\
\Delta_{g} f & =\frac{2(n-2)}{r^{2}}+O\left(r^{n-4}\right) \\
|\nabla f|_{g}^{2} & =\frac{4}{r^{2}}+O\left(r^{n-4}\right) .
\end{aligned}
$$

Since $g$ is flat near $p$, $\operatorname{Ric}_{g}$ vanishes and $g=d r^{2}+r^{2} \sigma^{n-1}$ where $\sigma^{n-1}$ stands for the usual metric on the standard sphere $\mathbb{S}^{n-1}$. We deduce from these computations that

$$
\begin{aligned}
\operatorname{Ric}_{g^{\prime}}= & -(n-2) b-\frac{2(n-2)}{r^{2}} d r^{2}+\frac{4(n-2)}{r^{2}} d r^{2}+ \\
& \left(\frac{2(n-2)}{r^{2}}-\frac{4(n-2)}{r^{2}}+O\left(r^{n-4}\right)\right)\left(d r^{2}+r^{2} \sigma^{n-1}\right)+\bar{O}\left(r^{n-4}\right) \\
= & -(n-2) b-2(n-2) \sigma^{n-1}+\bar{O}\left(r^{n-4}\right) .
\end{aligned}
$$

We get from (3), (6) and the definition of $H^{\prime}$ that on $S_{\epsilon}$

$$
\begin{aligned}
\operatorname{grad}^{g^{\prime}} H^{\prime} & =\Gamma^{-\frac{4}{n-2}} \operatorname{grad}^{g}\left(1+O\left(r^{n-4}\right)\right) \\
& =O\left(r^{n-1}\right) \frac{\partial}{\partial r}+v
\end{aligned}
$$

is a vector field such that $\operatorname{Ric}_{g^{\prime}}\left(v, \nu^{\prime}\right)=0$. Observe that by (13) and (18), we have $\sigma^{n-1}\left(\cdot, \nu^{\prime}\right)=0$ and $b\left(\cdot, \nu^{\prime}\right)=0$ on $S_{\epsilon}$. In addition, the estimates (13), (18) then imply that on $S_{\epsilon}$

$$
\begin{aligned}
\operatorname{Ric}_{g^{\prime}}\left(\operatorname{grad}^{g^{\prime}} H^{\prime}, \nu^{\prime}\right) & =\bar{O}\left(r^{n-4}\right)\left(\operatorname{grad}^{g^{\prime}} H^{\prime}, \nu^{\prime}\right) \\
& =O\left(\epsilon^{2 n-3}\right) .
\end{aligned}
$$

Relation (14) then leads to

$$
\mathbf{I I}=O\left(\epsilon^{n-2}\right)=o(1) .
$$

2.3. Conclusion. Using (8), (9), (15), (20) and passing to the limit $\epsilon \rightarrow 0$, we obtain that

$$
0=2(n-2)(n-4) \omega_{n-1} B-\frac{1}{2} \int_{M \backslash\{p\}}\left|E_{g^{\prime}}\right|^{2} H^{\prime} d v_{g^{\prime}} .
$$

Assumption (e) implies that $H^{\prime}>0$ and hence $B \geq 0$. This proves first part of Theorem 1.1.

Now, assume that $B=0$. Then $E_{g^{\prime}} \equiv 0$ on $M \backslash\{p\}$. This implies that $\left(M \backslash\{p\}, g^{\prime}\right)$ is Einstein and scalar flat hence Ricci flat. Since in addition the Weyl curvature is zero, $\left(M \backslash\{p\}, g^{\prime}\right)$ turns to be flat (see He97 p. 123). It is known that $\left(M \backslash\{p\}, g^{\prime}\right)$ is asymptotically flat and that its mass satisfies $m\left(g^{\prime}\right)=c_{n} C$ where $c_{n}>0$ (see e.g. Lee-Parker [LP87]). Since $g^{\prime}$ is flat, $m\left(g^{\prime}\right)=0$ so is $C$ and by a positive mass Theorem by Schoen-Yau [SY88, $(M, g)$ is conformally diffeomorphic to $\left(S^{n}, g\right)$.

Remark 2.1. It is clear from the proof that Assumption (a) can be weakened and replaced by

(a) $g$ is locally flat around a point $p$ and the standard Positive Mass Theorem is valid on $M$ (i.e. with the notations of Section 2, $C \geq 0$ with equality if and only 
if $(M, g)$ is conformally diffeomorphic to $\left.\mathbb{S}^{n}\right)$. In particular, by [SY79] and [AH03, this assumption holds if $n \in\{5,6,7\}$ or if $M$ is spin.

\section{REFERENCES}

[AH03] B. Ammann and E. Humbert, Positive mass theorem for the Yamabe problem on spin manifolds, Geom. and Func. Anal., 15 (2005), No 3, (567-576).

[Au98] T. Aubin, Some nonlinear problems in Riemannian geometry, Springer Monographs in Mathematics, Springer-Verlag, Berlin, 1998.

[Br87] T.P. Branson, Group representations arising from Lorentz conformal geometry, J. Func. Anal. 74 (1987), (199-291).

[GR07] H.C. Grunau and F. Robert, Positivity issues of biharmonic Green's functions under Dirichlet boundary conditions, Preprint 2007.

[He97] E. Hebey, Introduction à l'analyse non-linéaire sur les variétés, Diderot Éditeur, Arts et sciences., 1997.

[HR04] E. Hebey and F. Robert, Compactness and global estimates for the geometric Paneitz equation in high dimensions, Electronic Res. Announc. of the AMS, 10 (2004), (135141).

[LP87] J. M. Lee and T. H. Parker. The Yamabe problem, Bull. Am. Math. Soc., New Ser., 17 37-91, 1987.

[Pa83] S. Paneitz, A quartic conformally covariant differential operator for arbitrary pseudoRiemannian manifolds, Preprint 1983

[QR06a] J. Qing and D. Raske, Compactness for conformal metrics with constant Q-curvature on locally conformally flat manifolds, Calc. of Variations, 26 (2006), No3, (343-356).

[QR06b] J. Qing and D. Raske, On positive solutions to semilinear conformally invariant equations on locally conformally flat manifolds, Int. Math. Res. Not., 6 (2006), Art. ID 94172.

[SY79] R. Schoen and S.-T. Yau. On the proof of the positive mass conjecture in general relativity, Comm. Math. Phys., 65 (1979), (45-76).

[SY88] R. Schoen and S.-T. Yau. Conformally flat manifolds, Kleinian groups and scalar curvature, Invent. Math., 92 (1988), (47-71).

[Ra07] S. Raulot, Green functions for the Dirac operator under local boundary conditions and applications, Preprint arXiv:math/0703197/ 1

Institut Élie Cartan, BP 239, Université De NANCy 1, 54506 VAndoeuvre-LÈs-Nancy Cedex, France

E-mail address: humbert@iecn.u-nancy.fr

Institut de Mathématiques, Université de Neuchâtel, Rue Emile-Argand 11, 2007 NeUChÂTEL, SUISSE

E-mail address: simon.raulot@unine.ch 